\title{
A Mathematical Model for the Use of Energy Resources: A Singular Parabolic Equation
}

\section{Daniel López-García and Rosa Pardo}

\author{
Departamento de Análisis Matemático y Matemática Aplicada, Universidad \\ Complutense de Madrid \\ 28040, Madrid, Spain \\ E-mail(corresp.): rpardo@ucm.es \\ E-mail: dlopez03@ucm.es
}

Received March 29, 2019; revised November 11, 2019; accepted November 11, 2019

\begin{abstract}
We consider a singular parabolic equation $t^{\beta} u_{t}-\Delta u=f$, for $(x, t) \in$ $\Omega \times(0, T)$, arising in symmetric boundary layer flows. Here $\Omega \subset \mathbb{R}^{N}$ is a bounded domain with $C^{2}$ boundary $\partial \Omega, \beta \leq 1, f=f(t, x)$ is bounded, and $T>0$ is some fixed time. We establish sufficient conditions for the existence and uniqueness of a weak solution of this singular parabolic equation with Dirichlet boundary conditions, and we investigate its regularity.

There are two different cases depending on $\beta$. If $\beta<1$, for any initial data $u_{0} \in L^{2}(\Omega)$, there exists a unique weak solution, which in fact is a strong solution. The singularity is removable when $\beta<1$. While if $\beta=1$, there exists a unique solution of the singular parabolic problem $t u_{t}-\Delta u=f$. The initial data cannot be arbitrarily chosen. In fact, if $f$ is continuous and $f(t) \rightarrow f_{0}$, as $t \rightarrow 0$, then, this solution converges, as $t \rightarrow 0$, to the solution of the elliptic problem $-\Delta u=f_{0}$, for $x \in \Omega$, with Dirichlet boundary conditions. Hence, no initial data can be prescribed when $\beta=1$, and the singularity in that case is strong.
\end{abstract}

Keywords: singular parabolic equation, degenerate parabolic equations, existence, uniqueness, symmetric boundary layer, regularity.

AMS Subject Classification: 35K67; 35K20; 35K65; 76N10.

\section{Introduction}

The main goal of this paper is to solve a singular linear heat equation with a coefficient of the time derivative depending on $t$, and Dirichlet boundary conditions. Specifically, given $f=f(x, t)$ and some $u_{0}(x)$ to be prescribed

Copyright (c) 2020 The Author(s). Published by VGTU Press

This is an Open Access article distributed under the terms of the Creative Commons Attribution License (http://creativecommons.org/licenses/by/4.0/), which permits unrestricted use, distribution, and reproduction in any medium, provided the original author and source are credited. 
later, let us consider the following singular parabolic equation

$$
\left\{\begin{aligned}
t^{\beta} u_{t}-\Delta u & =f, & & (x, t) \in \Omega \times(0, T), \\
u(x, t) & =0, & & (x, t) \in \partial \Omega \times(0, T), \\
u(x, 0) & =u_{0}(x), & & x \in \Omega,
\end{aligned}\right.
$$

where $\Omega \subset \mathbb{R}^{N}$ is a bounded domain with $C^{2}$ boundary $\partial \Omega,-\infty<\beta \leq 1$ and $T>0$ is some fixed time. The parabolic equation (1.1) is singular at $t=0$.

Let us fix $\beta<1$. We introduce a new 'time' variable $s(=s(\beta, t))$,

$$
s:=\frac{1}{1-\beta} t^{1-\beta}, \quad \text { then } \frac{d s}{d t}=t^{-\beta}, \text { and } t=[(1-\beta) s]^{\frac{1}{1-\beta}} .
$$

As $t$ moves in $(0, T), s$ ranges between 0 and $S$, with

$$
S=s(\beta, T):=T^{1-\beta} /(1-\beta) .
$$

Let $u$ be a weak solution of the parabolic problem (1.1). The function $v(x, s):=u\left(x,[(1-\beta) s]^{\frac{1}{1-\beta}}\right)$, is a solution of

$$
\begin{cases}v_{s}-\Delta v=g, & (x, s) \in \Omega \times(0, S), \\ v(x, s)=0, & (x, s) \in \partial \Omega \times(0, S), \\ v(x, 0)=u_{0}(x), & x \in \Omega\end{cases}
$$

where

$$
g(x, s):=f\left(x,[(1-\beta) s]^{\frac{1}{1-\beta}}\right) .
$$

Equation (1.4) is a standard parabolic problem, accordingly there exists a unique weak solution of (1.4) (see, for instance, $[5,8,13,22,25]$ ), given by the formula of variation of constants (see (4.1)). Recovering variable $t$, we obtain that the problem (1.1) has a unique weak solution given by

$$
\begin{aligned}
u(t)= & \sum_{i=1}^{\infty} e^{-\frac{\lambda_{i}}{1-\beta} t^{1-\beta}}\left\langle u_{0}, \varphi_{i}\right\rangle \varphi_{i} \\
& +\sum_{i=1}^{\infty}\left(\int_{0}^{t} e^{-\frac{\lambda_{i}}{1-\beta}\left(t^{1-\beta}-\tau^{1-\beta}\right)} \tau^{-\beta}\left\langle f(\tau), \varphi_{i}\right\rangle d s\right) \varphi_{i},
\end{aligned}
$$

where $\langle\cdot, \cdot\rangle$ is the usual scalar product in $L^{2}(\Omega)$ and $\left\{\lambda_{i}\right\},\left\{\varphi_{i}\right\}$ are the eigenvalues and eigenfunctions (normalized in the $L^{2}$-norm) of the Dirichlet eigenvalue problem $-\Delta \varphi=\lambda \varphi, x \in \Omega, \varphi=0, x \in \partial \Omega$. In Theorems 3 and 4 we analyze the regularity of the solution, which is not the usual one.

Once known the case $\beta<1$, we analyze what happens when $\beta \rightarrow 1$. Obviously, the decomposition (1.6) becomes singular as $\beta \rightarrow 1$. Let us fix any initial time $t_{1}>0$. Instead of (1.1), given any initial data $\left(t_{1}, u_{1}\right)$, we consider the following initial value problem

$$
\left\{\begin{aligned}
t u_{t}-\Delta u & =f(x, t), & & (x, t) \in \Omega \times\left(t_{1}, T\right), \\
u(x, t) & =0, & & (x, t) \in \partial \Omega \times\left(t_{1}, T\right), \\
u\left(x, t_{1}\right) & =u_{1}(x), & & x \in \Omega .
\end{aligned}\right.
$$


We next reconsider the time variable $s=s(\beta, t)$ introduced in (1.2). To do that, set $s_{1}=s\left(\beta, t_{1}\right):=\frac{1}{1-\beta} t_{1}^{1-\beta}$, and introduce a new time variable $\theta:=$ $\lim _{\beta \rightarrow 1}\left[s(\beta, t)-s\left(\beta, t_{1}\right)\right]$, obtaining

$$
\theta=\ln \left(\frac{t}{t_{1}}\right), \quad \frac{d \theta}{d t}=\frac{1}{t}, \quad \text { and } t=t_{1} e^{\theta} .
$$

Obviously, as $t$ moves in $\left(t_{1}, T\right)$, the new variable $\theta$ ranges between $0\left(=\ln \left(t_{1} / t_{1}\right)\right)$ and

$$
\Theta=\Theta(T):=\ln \left(T / t_{1}\right) .
$$

Observe that, for any $t>0$ fixed, $\lim _{t_{1} \rightarrow 0} \ln \left(\frac{t}{t_{1}}\right)=-\infty$.

Reasoning as above, (when $\beta<1$ ), if $u$ is a weak solution of the parabolic problem $(1.7)_{t_{1}}$, then the function $v(x, \theta):=u\left(x, t_{1} e^{\theta}\right)$, is a solution of

$$
\left\{\begin{aligned}
v_{\theta}-\Delta v & =g, & & (x, \theta) \in \Omega \times(0, \Theta), \\
v(x, \theta) & =0, & & (x, \theta) \in \partial \Omega \times(0, \Theta), \\
v(x, 0) & =u_{1}(x), & & x \in \Omega,
\end{aligned}\right.
$$

where

$$
g(x, \theta):=f\left(x, t_{1} e^{\theta}\right) .
$$

Recovering variable $t=t_{1} e^{\theta}$, we obtain that the solution to the equation $(1.7)_{t_{1}}$ $u(t)=u\left(t ; t_{1}, u_{1}\right)$, through the variation of constants formulae, is given by

$$
u(t)=\sum_{i=1}^{\infty}\left(\frac{t_{1}}{t}\right)^{\lambda_{i}}\left\langle u_{1}, \varphi_{i}\right\rangle \varphi_{i}+\sum_{i=1}^{\infty} t^{-\lambda_{i}}\left(\int_{t_{1}}^{t} \sigma^{\lambda_{i}-1}\left\langle f(\sigma), \varphi_{i}\right\rangle d \sigma\right) \varphi_{i} .
$$

Assume that $t>0$ is fixed. Roughly speaking, letting $t_{1} \rightarrow 0$ we obtain that $\lim _{t_{1} \rightarrow 0} u\left(t ; t_{1}, u_{1}\right)=\mathbf{u}(t)$ where

$$
\mathbf{u}(t):=\sum_{i=1}^{\infty} t^{-\lambda_{i}}\left(\int_{0}^{t} \sigma^{\lambda_{i}-1}\left\langle f(\sigma), \varphi_{i}\right\rangle d \sigma\right) \varphi_{i},
$$

or, changing the integration variable,

$$
\mathbf{u}(t)=\sum_{i=1}^{\infty}\left(\int_{0}^{1}\left\langle f(t \tau), \varphi_{i}\right\rangle \tau^{\lambda_{i}-1} d \tau\right) \varphi_{i}
$$

and the initial data $u_{1}$ can not be arbitrarily chosen as $t_{1} \rightarrow 0$. The initial value problem $(1.7)_{t_{1}}$ loses its initial condition as $t_{1} \rightarrow 0$, and it is reduced to

$$
t u_{t}-\Delta u=f(x, t), \quad \text { in } \Omega \times(0, T), \quad u(x, t)=0, \quad \text { on } \partial \Omega \times(0, T) .
$$

Moreover, roughly speaking, if $f(t, x) \rightarrow f_{0}(x)$ when $t \rightarrow 0$, then

$$
\mathbf{u}(t) \rightarrow \mathbf{u}(0):=\sum_{i=1}^{\infty} \frac{1}{\lambda_{i}}\left\langle f_{0}, \varphi_{i}\right\rangle \varphi_{i} .
$$


Henceforth, the unprescribed initial data in equation (1.15), coincides with the solution of the Dirichlet problem

$$
-\Delta u=f_{0}, \quad x \in \Omega, \quad u=0, \quad x \in \partial \Omega .
$$

As a conclusion, problem (1.1) for $\beta=1$ and a general initial data $u_{0}$ is ill posed. In Theorems 7 and 8 we provide sufficient conditions on the source term $f$ for the existence of a unique weak solution of (1.15) and we also analyze its corresponding regularity, which again is not the usual one.

Singular parabolic problems arise in a lot of fields of technology and natural sciences. These problems may be divided on different classes accordingly to their singularity with respect either to spatial variables $[9,12,16,26]$, to time variable $[3,14,17]$, or to the unknown $u$, or its gradient, see $[1,2,4,7,10,15,18$, $23,24,27,28]$.

Equation (1.1) arises in symmetric boundary layer flows. Let $u$ be the temperature for steady boundary layer flows around a blunt body. The governing equation can be written in the form

$$
\begin{cases}\rho c_{p} w u_{t}-\lambda u_{z z}=f\left(z, t, u, u_{z}\right), & t>0, \quad z \in(0, l), \\ u(t, 0)=u_{1}(t), \quad u(t, l)=u_{l}(t), & \text { for } t \geq 0, \\ u(0, z)=u_{0}(z), & \end{cases}
$$

where $\rho$ denotes the gas density, $c_{p}$ the gas heat capacity, $w$ the tangential velocity component, $t$ a curvilinear coordinate measured along the body surface, $z$ the coordinate normal to the body surface, $\lambda$ the gas thermal conductivity and where $f$ contains the lower order derivatives with respect to the normal coordinate $z$, see [11, equation (2.13)]. For usual boundary layer flows, the tangential velocity component $w$ is positive in the interior of the domain, which guarantees the standard parabolic aspect of (1.17). However, for symmetric boundary layers, the tangential velocity $w$ depends on $t$, and vanishes at $t=0$ $(w(0)=0)$, in such a way that there is a singularity in the initial value problem with respect to $t$.

Moreover, similar equations appear in energy resources and energy consumption problems. Nowadays, the society demands a lot of energy consumption. Fossil fuels overuse and effects their applications have caused, make the study about green, renewable resources a priority. Mathematical models play an important role on this development, trying to get insight in how the energy sources work. On one hand, almost all the systems to produce energy use fossil fuels, biomass or nuclear fuels to heat a fluid. After that, the fluid access a turbine to work producing electricity subsequently. On the other hand, there is a very solid technology that uses the solar resource, both directly or indirectly, to produce electricity: solar power towers, photoelectric panels, wind farms or other devices based on the water cycle. Related problems to (1.1) appear modeling the use of salts in thermosolar power towers, and also in molten salt reactors. In both models, a molten salt is heated by using solar energy or nuclear reactions respectively. The temperature rises up between 500 and $1000^{\circ} \mathrm{C}$, depending on the model, although not regularly in the first place. Until the salt completely heats, there are different zones where the salt is hotter than others. That creates density differences that leads to symmetric boundary layers. 
The understanding of this phenomena is directly related with the efficiency of the salts to collect energy and avoid energy loss. For more information about the processes involved in molten salt reactors and solar power towers, see [21] and [6] respectively.

The case $\beta=1$ is a limit case appearing in symmetric boundary layers like (1.17), when the tangential velocity $w(0)=0$, it is is such that $w /(t z)$ remains positive for $t>0$, and $0<z<l$, so that the underlying operator is essentially $t(\partial / \partial t)-(1 / z)\left(\partial^{2} / \partial z^{2}\right)$, or more generally, $t(\partial / \partial t)+L$, where $L$ is a partial differential operator with respect to the normal coordinate. In this situation, no initial conditions with respect to $t$ can be prescribed, due to the singularity, and the temperature on the symmetry axis have to be obtained formally by letting $t=0$ in the PDE contained in (1.17). This singularity also appears with the velocity governing equations in the case of incompressible boundary layer flows. An existence result of Oleinik is available, see [20]. The flow equations, after a suitable change of variable, can be reduced to the equation

$$
\begin{cases}t v_{t}-\frac{\nu(v)}{z} v_{z z}=f\left(z, t, v, v_{z}\right), & s \geq 0, \quad 0 \leq z \leq 1, \\ v(t, 0)=v_{1}(t), \quad v(t, l)=v_{2}(t), & \text { for } \quad s \geq 0,\end{cases}
$$

where $(z, t)$ are rescaled tangential and normal coordinates to the body surface, and $v$ is a rescaled normal derivative of the tangential velocity component $w$, see [20]. Since the same type of singularity appears for $t=0$ as for the temperature equation in compressible flows, no initial condition is to be prescribed for $t=0$, see [20], and this is the main difference with the non symmetric boundary layer case where the tangential velocity $w$ remains positive inside the domain, see $[19]$.

This paper is organized in the following way. Section 2 contains preliminaries, and well known results, in order to reformulate problem (1.1) in a weak framework. In Section 3 we state our main results. In Section 4 we study a standard parabolic problem (1.4), (related with problem $(1.1)$ when $\beta<1$ ). Section 5 considers the case $\beta<1$. Section 6 covers the case $\beta=1, t_{1}>0$. In Section 7 letting $t_{1} \rightarrow 0$, we consider the singular problem (1.15) with $\beta=1$.

\section{Preliminaries, known results, and weak formulation}

This section contains well known concepts, in order to precise a weak formulation of (1.1). Let us consider the Hilbert spaces $L^{2}(\Omega)$ and $H_{0}^{1}(\Omega)$ with their usual scalar product and norms

$$
\begin{aligned}
\langle u, v\rangle:=\int_{\Omega} u(x) v(x) d x, \quad\|v\|_{L^{2}(\Omega)}:=\left(\int_{\Omega}|v|^{2} d x\right)^{1 / 2}, \\
(u, v):=\int_{\Omega} \nabla u(x) \cdot \nabla v(x) d x, \quad\|v\|_{H_{0}^{1}(\Omega)}:=\left(\int_{\Omega}|\nabla v(x)|^{2} d x\right)^{1 / 2} .
\end{aligned}
$$

Let us consider the dual space of $H_{0}^{1}(\Omega)$ denoted by $H^{-1}(\Omega), f \in H^{-1}(\Omega)$ if $f$ is a bounded linear functional on $H_{0}^{1}(\Omega)$. We denote the duality product of $H^{-1}(\Omega)$ in $H_{0}^{1}(\Omega)$ by $\langle\cdot, \cdot\rangle_{H^{-1}(\Omega), H_{0}^{1}(\Omega)}$. We define the norm

$$
\|f\|_{H^{-1}(\Omega)}:=\sup _{v \in H_{0}^{1}(\Omega)}\left\{\langle f, v\rangle_{H^{-1}(\Omega), H_{0}^{1}(\Omega)}:\|v\|_{H_{0}^{1}(\Omega)} \leq 1\right\} .
$$


A characterization of $H^{-1}(\Omega)$ and its norm is given by the following theorem, see [8, Theorem 1 in $\S 5.9 .1]$.

Theorem 1. The function $f \in H^{-1}(\Omega)$ if and only if there exist functions $f_{i} \in L^{2}(\Omega), i=0,1, \ldots, N$ such that

$$
\langle f, v\rangle_{H^{-1}(\Omega), H_{0}^{1}(\Omega)}=\int_{\Omega} f_{0} v+\sum_{i=1}^{N} \int_{\Omega} f_{i} \frac{\partial v}{\partial x_{i}}, \quad \forall v \in H_{0}^{1}(\Omega) .
$$

Furthermore, given $f_{i} \in L^{2}(\Omega), i=0,1, \ldots, N$ satisfying (2.1), then

$$
\|f\|_{H^{-1}(\Omega)}=\inf \left\{\left(\int_{\Omega} \sum_{i=0}^{N}\left|f_{i}\right|^{2} d x\right)^{1 / 2}\right\} .
$$

Fix $\beta<1$. Let us assume that $u(x, t)$ is a solution of (1.1) regular enough. Multiplying (1.1) by an arbitrary test function $\phi \in H_{0}^{1}(\Omega)$, and integrating over $\Omega$,

$$
t^{\beta} \frac{d}{d t} \int_{\Omega} u(x, t) \phi(x) d x+\int_{\Omega} \nabla u(x, t) \cdot \nabla \phi(x) d x=\int_{\Omega} f(x, t) \phi(x) d x,
$$

for all $\phi \in H_{0}^{1}(\Omega)$. We associate with $u$ a mapping $\mathbf{u}:[0, T] \rightarrow H_{0}^{1}(\Omega)$ defined by $[\mathbf{u}(t)](x):=u(x, t)$. We consider $u$ not as a function of $x$, and $t$, but instead as a mapping $\mathbf{u}$ of $t$ into the space $H_{0}^{1}(\Omega)$. Similarly, we define $\mathbf{f}:[0, T] \rightarrow$ $L^{2}(\Omega)$. The singular heat equation (1.1) can be rewritten as follows: find a function $\mathbf{u}: t \in[0, T] \longrightarrow \mathbf{u}(t) \in H_{0}^{1}(\Omega)$ such that $\forall \phi \in H_{0}^{1}(\Omega), \forall t \in(0, T)$ :

$$
t^{\beta} \frac{d}{d t}\langle\mathbf{u}(t), \phi\rangle+a(\mathbf{u}(t), \phi)=\langle\mathbf{f}(t), \phi\rangle ; \quad \mathbf{u}(0)=u_{0},
$$

where $a$ is a bilinear form defined by $a(u, v):=\int_{\Omega} \nabla u(x) \cdot \nabla v(x) d x, \forall u, v \in$ $H^{1}(\Omega)$, so

$$
a(\mathbf{u}(t), \phi)=\int_{\Omega} \nabla u(x, t) \cdot \nabla \phi(x) d x .
$$

Next, observe that

$$
t^{\beta} \frac{d}{d t} \mathbf{u}(t)=\mathbf{f}(t)+\operatorname{div} \nabla \mathbf{u}, \quad \text { a.e. in } \Omega \times[0, T] .
$$

The above right hand side lies in $H^{-1}(\Omega)$, see Theorem 1, with

$$
t^{\beta}\left\|\mathbf{u}_{t}(t)\right\|_{H^{-1}(\Omega)} \leq\|\mathbf{f}(t)\|_{L^{2}(\Omega)}+\|\mathbf{u}(t)\|_{H_{0}^{1}(\Omega)} .
$$

This estimate suggests to look for a weak solution with $t^{\beta} \frac{d}{d t} \mathbf{u}(t) \in H^{-1}(\Omega)$ for a. e. $t \in[0, T]$, in which case, the first term in (2.3) can be re-expressed as the duality pairing of $H^{-1}(\Omega)$ in $H_{0}^{1}(\Omega)$ by $\left\langle t^{\beta} \frac{d}{d t} \mathbf{u}(t), \phi\right\rangle_{H^{-1}(\Omega), H_{0}^{1}(\Omega)}$.

To define a weak solution, we introduce some Sobolev spaces involving time. Let $Y$ denote a Banach space with norm $\|\cdot\|_{Y}$. The space $L^{p}((0, T) ; Y)$, for $p \in[1, \infty)$, consists of all strongly measurable functions $\mathbf{u}:[0, T] \rightarrow Y$ with

$$
\|\mathbf{u}\|_{L^{p}((0, T) ; Y)}:=\left(\int_{0}^{T}\|\mathbf{u}(t)\|_{Y}^{p}\right)^{1 / p}<+\infty
$$


then the space $L^{\infty}((0, T) ; Y)$ comprises all strongly measurable functions $\mathbf{u}$ : $[0, T] \rightarrow Y$ with

$$
\|\mathbf{u}\|_{L^{\infty}((0, T) ; Y)}:=\operatorname{ess} \sup _{t \in[0, T]}\|\mathbf{u}(t)\|_{Y}<+\infty
$$

and the space $C((0, T) ; Y)$ consists of all continuous functions $\mathbf{u}:[0, T] \rightarrow Y$ with

$$
\|\mathbf{u}\|_{C((0, T) ; Y)}:=\max _{t \in[0, T]}\|\mathbf{u}(t)\|_{Y}<+\infty .
$$

The next theorem concerns what happens when $\mathbf{u}$ and $\mathbf{u}_{t}$ lie in different spaces, see [8, Theorem 3 in $\S 5.9 .2]$.

Theorem 2. Suppose $\mathbf{u} \in L^{2}\left((0, T) ; H_{0}^{1}(\Omega)\right)$ with $\mathbf{u}_{t} \in L^{2}\left((0, T) ; H^{-1}(\Omega)\right)$. Then, (after possibly being redefined on a set of zero measure), function $\mathbf{u} \in$ $C\left([0, T] ; L^{2}(\Omega)\right)$.

Definition 1. Let us fix $\beta<1$. Given $u_{0} \in L^{2}(\Omega)$ and $\mathbf{f} \in L^{\infty}\left((0, T) ; L^{2}(\Omega)\right)$, we say that a function $\mathbf{u} \in L^{2}\left((0, T) ; H_{0}^{1}(\Omega)\right) \cap C\left([0, T] ; L^{2}(\Omega)\right)$ with $t^{\beta} \mathbf{u}_{t} \in$ $L^{2}\left((0, T) ; H^{-1}(\Omega)\right)$, and $\mathbf{u}_{t} \in L^{1}\left((0, T) ; H^{-1}(\Omega)\right)$, is a weak solution of the parabolic problem (1.1) with $\beta<1$, provided (2.3) is satisfied.

Remark 1 . The usual definition of a weak solution in a standard parabolic problem $(\beta=0)$, gives $\mathbf{f} \in L^{2}\left((0, T) ; L^{2}(\Omega)\right)$ and asks for $\mathbf{u}_{t} \in L^{2}\left((0, T) ; H^{-1}(\Omega)\right)$. If the data $\mathbf{f} \in L^{2}\left((0, T) ; L^{2}(\Omega)\right)$, then the solution of (1.1) is not regular enough. From (2.4),

$$
\left\|\mathbf{u}_{t}(t)\right\|_{H^{-1}(\Omega)} \leq t^{-\beta}\|\mathbf{f}(t)\|_{L^{2}(\Omega)}+t^{-\beta}\|\mathbf{u}(t)\|_{H_{0}^{1}(\Omega)},
$$

and by definition of $\|\cdot\|_{L^{2}\left((0, T) ; H^{-1}(\Omega)\right)}$, see $(2.5)$,

$$
\begin{gathered}
\left\|\mathbf{u}_{t}\right\|_{L^{2}\left((0, T) ; H^{-1}(\Omega)\right)}^{2} \leq \int_{0}^{T} t^{-2 \beta}\left(\|\mathbf{f}(t)\|_{L^{2}(\Omega)}+\|\mathbf{u}(t)\|_{H_{0}^{1}(\Omega)}\right)^{2} d t \\
\leq 2 \int_{0}^{T} t^{-2 \beta}\left(\|\mathbf{f}(t)\|_{L^{2}(\Omega)}^{2}+\|\mathbf{u}(t)\|_{H_{0}^{1}(\Omega)}^{2}\right) d t
\end{gathered}
$$

Obviously, if $\mathbf{f} \in L^{\infty}\left((0, T) ; L^{2}(\Omega)\right)$, and $\beta<1 / 2$, then

$$
\int_{0}^{T} t^{-2 \beta}\|\mathbf{f}(t)\|_{L^{2}(\Omega)}^{2} d t \leq \frac{T^{1-2 \beta}}{1-2 \beta}\|\mathbf{f}\|_{L^{\infty}\left((0, T) ; L^{2}(\Omega)\right)}^{2} .
$$

This is not a completely satisfactory estimate, due to in one hand we would like to allow $\beta<1$, not to restrict $\beta<1 / 2$. But moreover, on the other hand, to be able to bound the second term on the right hand side of $(2.7)$, we need $\mathbf{u} \in L^{\infty}\left((0, T) ; H_{0}^{1}(\Omega)\right)$ (which is not a usual requirement for a weak solution), and again $\beta<1 / 2$. If instead, we look for un upper bound of $\left\|\mathbf{u}_{t}\right\|_{L^{1}\left((0, T) ; H^{-1}(\Omega)\right)}$ we deduce the following

$$
\left\|\mathbf{u}_{t}\right\|_{L^{1}\left((0, T) ; H^{-1}(\Omega)\right)} \leq \int_{0}^{T} t^{-\beta}\left(\|\mathbf{f}(t)\|_{L^{2}(\Omega)}+\|\mathbf{u}(t)\|_{H_{0}^{1}(\Omega)}\right) d t
$$


and if $\mathbf{f} \in L^{\infty}\left((0, T) ; L^{2}(\Omega)\right)$, and $\beta<1$, then

$$
\int_{0}^{T} t^{-\beta}\|\mathbf{f}(t)\|_{L^{2}(\Omega)} d t \leq \frac{T^{1-\beta}}{1-\beta}\|\mathbf{f}\|_{L^{\infty}\left((0, T) ; L^{2}(\Omega)\right)} .
$$

And again to bound the second term on the right hand side of (2.8), we need $\mathbf{u} \in L^{\infty}\left((0, T) ; H_{0}^{1}(\Omega)\right)$.

At this moment the hypothesis $t^{\beta} \mathbf{u}_{t} \in L^{2}\left((0, T) ; H^{-1}(\Omega)\right)$ looks like the natural one, but it is not immediate to deduce that $\mathbf{u}_{t} \in L^{1}\left((0, T) ; H^{-1}(\Omega)\right)$ when $\beta<1$. We will need sharper estimates.

We now concentrate our attention to the case $\beta=1$. Fixing $t_{1}>0$, we study the standard parabolic equation with arbitrary initial data $\left(t_{1}, u_{1}\right)$, given by $(1.7)_{t_{1}}$, and then let $t_{1} \rightarrow 0$. We state the definitions of weak solutions. The first definition is for $t_{1}>0$ fixed. Reasoning as in the introduction, the second definition is obtained letting $t_{1} \rightarrow 0$.

Definition 2. Given $t_{1}>0, u_{1} \in L^{2}(\Omega)$ and $\mathbf{f} \in L^{\infty}\left(\left(t_{1}, T\right) ; L^{2}(\Omega)\right)$, we say that a function $\mathbf{u}(t)=\mathbf{u}\left(t ; t_{1}, u_{1}\right), \mathbf{u} \in L^{2}\left(\left(t_{1}, T\right) ; H_{0}^{1}(\Omega)\right) \cap C\left(\left[t_{1}, T\right] ; L^{2}(\Omega)\right)$ with $t \mathbf{u}_{t} \in L^{2}\left(\left(t_{1}, T\right) ; H^{-1}(\Omega)\right)$, and $\mathbf{u}_{t} \in L^{1}\left(\left(t_{1}, T\right) ; H^{-1}(\Omega)\right)$, is a weak solution of $(1.7)_{t_{1}}$ provided

$$
t\left\langle\mathbf{u}_{t}(t), \phi\right\rangle+a(\mathbf{u}(t), \phi)=\langle\mathbf{f}(t), \phi\rangle, \quad \forall \phi \in H_{0}^{1}(\Omega), \forall t \in\left(t_{1}, T\right),
$$

is satisfied, and also $\mathbf{u}\left(t_{1}\right)=u_{1}$.

Remark 2. When $\beta=1$, an estimate like (2.9) is unreachable, hence in that case we can not wait for a solution with time derivative in $L^{1}\left((0, T) ; H^{-1}(\Omega)\right)$. We look instead for a solution such that $t \mathbf{u}_{t} \in L^{2}\left((0, T) ; H^{-1}(\Omega)\right)$.

Definition 3. Given $\mathbf{f} \in L^{\infty}\left((0, T) ; L^{2}(\Omega)\right)$, we say that a function $\mathbf{u}(t), \mathbf{u} \in$ $L^{2}\left((0, T) ; H_{0}^{1}(\Omega)\right) \cap C\left([0, T] ; L^{2}(\Omega)\right)$ with $t \mathbf{u}_{t} \in L^{2}\left((0, T) ; H^{-1}(\Omega)\right)$, is a weak solution of the parabolic problem (1.15) provided

$$
t\left\langle\mathbf{u}_{t}(t), \phi\right\rangle+a(\mathbf{u}(t), \phi)=\langle\mathbf{f}(t), \phi\rangle, \quad \forall \phi \in H_{0}^{1}(\Omega), \forall t \in(0, T),
$$

is satisfied.

\section{Main results}

In this Section 3 we state our main results, Theorems 3,4 (case $\beta<1$ ), Theorems 5,6 (case $\beta=1, t_{1}>0$ ), and Theorems 7,8 (case $\beta=1$ ).

The following two theorems provide a result on existence of a unique weak solution of (1.1), when $\mathbf{f} \in L^{\infty}\left((0, T) ; L^{2}(\Omega)\right)$ and $\beta<1$.

Theorem 3. Given $\mathbf{f} \in L^{\infty}\left((0, T) ; L^{2}(\Omega)\right)$ and $\beta<1$, for each $u_{0} \in L^{2}(\Omega)$, the problem (1.1) has a unique weak solution given by (1.6). Moreover, there exists a constant $C>0$, depending only on $\Omega, \beta$ and $T$, such that

$$
\begin{gathered}
\max _{t \in[0, T]}\|\mathbf{u}(t)\|_{L^{2}(\Omega)}+\|\mathbf{u}\|_{L^{2}\left((0, T) ; H_{0}^{1}(\Omega)\right)}+\left\|\mathbf{u}_{t}\right\|_{L^{1}\left((0, T) ; H^{-1}(\Omega)\right)} \\
\leq C\left(\left\|u_{0}\right\|_{L^{2}(\Omega)}+\|\mathbf{f}\|_{L^{\infty}\left((0, T) ; L^{2}(\Omega)\right)}\right) .
\end{gathered}
$$


And, if $\beta<1 / 2$, then $\mathbf{u}_{t} \in L^{2}\left((0, T) ; H^{-1}(\Omega)\right)$, and there exists a constant $C>0$ depending only on $\Omega, \beta$ and $T$, such that

$$
\left\|\mathbf{u}_{t}\right\|_{L^{2}\left((0, T) ; H^{-1}(\Omega)\right)} \leq C\left(\left\|u_{0}\right\|_{L^{2}(\Omega)}+\|\mathbf{f}\|_{L^{\infty}\left((0, T) ; L^{2}(\Omega)\right)}\right) .
$$

The regularity of a weak solution of (1.1) can be improved assuming that the boundary $\partial \Omega$ is regular enough.

Theorem 4. Given $u_{0} \in L^{2}(\Omega), \mathbf{f} \in L^{\infty}\left((0, T) ; L^{2}(\Omega)\right)$ and $\beta<1$, let $\mathbf{u}$ be the unique weak solution of the problem (1.1) given by (1.6). Then, in fact $\mathbf{u} \in L^{2}\left((0, T), H^{2}(\Omega)\right) \cap L^{\infty}\left((0, T) ; H_{0}^{1}(\Omega)\right)$, with $\mathbf{u}_{t} \in L^{1}\left((0, T) ; L^{2}(\Omega)\right)$, and for some constant $C>0$ (depending only on $\Omega, \beta$ and $T$ ), we have the estimate

$$
\begin{gathered}
\operatorname{ess} \sup _{t \in[0, T]}\|\mathbf{u}(t)\|_{H_{0}^{1}(\Omega)}+\|\mathbf{u}\|_{L^{2}\left((0, T) ; H^{2}(\Omega)\right)}+\left\|\mathbf{u}_{t}\right\|_{L^{1}\left((0, T) ; L^{2}(\Omega)\right)} \\
\leq C\left(\left\|u_{0}\right\|_{L^{2}(\Omega)}+\|\mathbf{f}\|_{L^{\infty}\left((0, T) ; L^{2}(\Omega)\right)}\right) .
\end{gathered}
$$

The following two theorems provide a result on the existence of a unique weak solution of the singular problem $(1.7)_{t_{1}}($ for $\beta=1)$.

Theorem 5. Given $t_{1}>0$, and $\mathbf{f} \in L^{\infty}\left((0, T) ; L^{2}(\Omega)\right)$, for each $u_{1} \in L^{2}(\Omega)$, the problem (1.7) $)_{t_{1}}$ has a unique weak solution given by (1.12). Moreover, there exists a constant $C>0$, depending only on $\Omega, \beta$ and $T$, such that

$$
\begin{gathered}
\max _{t \in\left[t_{1}, T\right]}\|\mathbf{u}(t)\|_{L^{2}(\Omega)}+\|\mathbf{u}\|_{L^{2}\left(\left(t_{1}, T\right) ; H_{0}^{1}(\Omega)\right)}+\left\|\mathbf{u}_{t}\right\|_{L^{1}\left(\left(t_{1}, T\right) ; H^{-1}(\Omega)\right)} \\
\leq C\left(\left\|u_{0}\right\|_{L^{2}(\Omega)}+\left[\ln \left(\frac{T}{t_{1}}\right)\right]^{1 / 2}\|\mathbf{f}\|_{L^{\infty}\left((0, T) ; L^{2}(\Omega)\right)}\right) .
\end{gathered}
$$

Assuming that the boundary $\partial \Omega$ is smooth enough, we improve the regularity of a weak solution of $(1.7)_{t_{1}}$.

Theorem 6. Given $t_{1}>0, u_{1} \in L^{2}(\Omega)$, and $\mathbf{f} \in L^{\infty}\left((0, T) ; L^{2}(\Omega)\right)$, let $\mathbf{u}$ be the unique weak solution of the problem (1.7) $t_{t_{1}}$ given by (1.12). Then, in fact $\mathbf{u} \in L^{2}\left(\left(t_{1}, T\right), H^{2}(\Omega)\right) \cap C\left(\left[t_{1}, T\right] ; H_{0}^{1}(\Omega)\right)$, with $\mathbf{u}_{t} \in L^{1}\left(\left(t_{1}, T\right) ; L^{2}(\Omega)\right)$, and there exists some constant $C>0$ depending only on $\Omega$ and $T$, such that

$$
\begin{gathered}
\operatorname{ess} \sup _{t \in[0, T]}\|\mathbf{u}(t)\|_{H_{0}^{1}(\Omega)}+\|\mathbf{u}\|_{L^{2}\left((0, T) ; H^{2}(\Omega)\right)}+\left\|\mathbf{u}_{t}\right\|_{L^{1}\left((0, T) ; L^{2}(\Omega)\right)} \\
\leq C\left(\left\|u_{0}\right\|_{L^{2}(\Omega)}+\left[\ln \left(T / t_{1}\right)\right]^{1 / 2}\|\mathbf{f}\|_{L^{\infty}\left((0, T) ; L^{2}(\Omega)\right)}\right) .
\end{gathered}
$$

Next we let $t_{1} \rightarrow 0$. The following two theorems provide a result on the existence of a unique weak solution of the singular problem (1.15) (for $\beta=1$ ). Obviously, estimates (3.1)-(3.2) do not apply when $t_{1} \rightarrow 0$. We have to restrict the space for the functions $\mathbf{f}$. Let $\mathbf{X}\left(=\mathbf{X}_{[0, T]}\right)$ be a Hilbert space defined by:

$$
\mathbf{X}:=\left\{\mathbf{f} \in L^{2}\left([0, T] ; L^{2}(\Omega)\right):\|\mathbf{f}\|_{\mathbf{X}}<+\infty\right\},
$$

where its associated norm is defined by:

$$
\|\mathbf{f}\|_{\mathbf{X}}:=\left[\int_{0}^{T}\left(\sum_{i=1}^{\infty} \sup _{\sigma \in[0, t]}\left|\left\langle\mathbf{f}(\sigma), \varphi_{i}\right\rangle\right|^{2}\right) d t\right]^{1 / 2}
$$


Theorem 7. Assume that $\mathbf{f} \in \mathbf{X} \cap C\left([0, T] ; L^{2}(\Omega)\right)$. Assume further that there exists two constant $C>0, \delta>0$ such that

$$
\sum_{i=1}^{\infty} \sup _{\sigma \in[0, t]}\left|\left\langle\mathbf{f}(\sigma), \varphi_{i}\right\rangle\right|^{2} \leq C, \text { for all } t \leq \delta .
$$

Then, the following holds:

(i) The problem (1.15) has a unique weak solution $\mathbf{u} \in L^{2}\left((0, T) ; H_{0}^{1}(\Omega)\right)$ with $t \mathbf{u}_{t} \in L^{2}\left((0, T) ; H^{-1}(\Omega)\right)$, given by the formula of variation of constants (1.13) or equivalently (1.14).

(ii) Moreover, there exists a constant $C>0$, depending only on $\Omega$, and $T$, such that

$$
\|\mathbf{u}\|_{L^{2}\left((0, T) ; H_{0}^{1}(\Omega)\right)}+\left\|t \mathbf{u}_{t}\right\|_{L^{2}\left((0, T) ; H^{-1}(\Omega)\right)} \leq C\|\mathbf{f}\|_{X}
$$

Theorem 8. Assume that $\mathbf{f} \in \mathbf{X} \cap C\left([0, T] ; L^{2}(\Omega)\right)$. Let $\mathbf{u}$ be the unique weak solution of the problem (1.15) given by (1.13). Then $\mathbf{u} \in L^{2}\left((0, T), H^{2}(\Omega)\right)$, with $t \mathbf{u}_{t} \in L^{2}\left((0, T) ; L^{2}(\Omega)\right)$, and we have the estimate

$$
\|\mathbf{u}\|_{L^{2}\left((0, T) ; H^{2}(\Omega)\right)}+\left\|t \mathbf{u}_{t}\right\|_{L^{2}\left((0, T) ; L^{2}(\Omega)\right)} \leq C\|\mathbf{f}\|_{\mathbf{X}}
$$

for some constant $C>0$ depending only on $\Omega$ and $T$. Furthermore, if

$$
\sum_{i=1}^{\infty} \sup _{\sigma \in[0, t]}\left|\left\langle\mathbf{f}(\sigma), \varphi_{i}\right\rangle\right|^{2} \leq C, \quad \text { for all } t \in(0, T] .
$$

then, $\mathbf{u} \in C\left((0, T] ; L^{2}(\Omega)\right)$. Moreover, if

$$
\sum_{i=1}^{\infty} \sup _{\sigma \in[0, t]}\left|\left\langle\mathbf{f}(\sigma)-\mathbf{f}(0), \varphi_{i}\right\rangle\right|^{2} \rightarrow 0 \quad \text { as } t \rightarrow 0
$$

then, there exists the limit $\lim _{t \rightarrow 0} \mathbf{u}(t)=\mathbf{u}(0)$ in $H^{2}(\Omega)$, and $\mathbf{u}(0) \in H^{2}(\Omega)$ is the unique solution of the Dirichlet problem

$$
-\Delta \mathbf{u}(0)=\mathbf{f}(0), \quad x \in \Omega, \quad u=0, \quad x \in \partial \Omega .
$$

\section{The standard parabolic problem}

This section collects well known results for a standard parabolic problem. The following theorem is a well known result on existence of a weak solution $\mathbf{v}(s)(\cdot):=v(s, \cdot)$ of the standard parabolic problem $(1.4)$, for $\mathbf{g}(s)(\cdot):=g(s, \cdot)$, and $S$ defined by $(1.3)$, see for instance $[5,8,13,22,25]$.

Theorem 9. Assume that $\mathbf{g} \in L^{2}\left((0, S) ; L^{2}(\Omega)\right)$. Then for every $u_{0} \in L^{2}(\Omega)$, there is a unique weak solution of problem (1.4), 
$\mathbf{v} \in L^{2}\left((0, S) ; H_{0}^{1}(\Omega)\right) \cap C\left([0, S] ; L^{2}(\Omega)\right)$, with $\mathbf{v}_{s} \in L^{2}\left((0, S) ; H^{-1}(\Omega)\right)$, given by the formula of variation of constants, that is

$$
\mathbf{v}(s)=\sum_{i=1}^{\infty} e^{-\lambda_{i} s}\left\langle u_{0}, \varphi_{i}\right\rangle \varphi_{i}+\sum_{i=1}^{\infty}\left(\int_{0}^{s} e^{-\lambda_{i}(s-\sigma)}\left\langle\mathbf{g}(\sigma), \varphi_{i}\right\rangle d \sigma\right) \varphi_{i} .
$$

Moreover, there exists a constant $C>0$ depending only on $\Omega$ and $S$, such that

$$
\begin{aligned}
\max _{s \in[0, S]} & \|\mathbf{v}(s)\|_{L^{2}(\Omega)}+\|\mathbf{v}\|_{L^{2}\left((0, S) ; H_{0}^{1}(\Omega)\right)}+\left\|\mathbf{v}_{s}\right\|_{L^{2}\left((0, S) ; H^{-1}(\Omega)\right)} \\
& \leq C\left(\left\|u_{0}\right\|_{L^{2}(\Omega)}+\|\mathbf{g}\|_{L^{2}\left((0, S) ; L^{2}(\Omega)\right)}\right) .
\end{aligned}
$$

Proof. From [8, Theorems 3 and 4 in $\S 7.1 .2]$, there exists a unique weak solution of (1.4), $v \in L^{2}\left((0, S) ; H_{0}^{1}(\Omega)\right)$, with $v_{s} \in L^{2}\left((0, S) ; H^{-1}(\Omega)\right)$. Thanks to Theorem 2.5, see also [8, Theorem 3 in $\S 5.9 .2]$, the solution is in $C\left([0, S] ; L^{2}(\Omega)\right)$, and so the initial condition makes sense. From $[8$, Theorem 2 in $§ 7.1 .2]$, we deduce the estimates (4.2).

The following theorem is a well known result improving the regularity of a weak solution $\mathbf{v}$ of the standard parabolic problem (1.4).

Theorem 10. Assume that $\mathbf{g} \in L^{2}\left((0, S) ; L^{2}(\Omega)\right)$. For every $u_{0} \in L^{2}(\Omega)$, let $\mathbf{v}$ be the unique weak solution of (1.4), given by the formula of variation of constants (4.1). Then, in fact $\mathbf{v} \in L^{2}\left((0, S), H^{2}(\Omega)\right) \cap L^{\infty}\left((0, S) ; H_{0}^{1}(\Omega)\right)$, with $\mathbf{v}_{s} \in L^{2}\left((0, S) ; L^{2}(\Omega)\right)$, and there exists a constant $C>0$ depending only on $\Omega$ and $S$, such that

$$
\begin{aligned}
& \text { ess } \sup _{s \in[0, S]}\|\mathbf{v}(s)\|_{H_{0}^{1}(\Omega)}+\|\mathbf{v}\|_{L^{2}\left((0, S) ; H^{2}(\Omega)\right)}+\left\|\mathbf{v}_{s}\right\|_{L^{2}\left((0, S) ; L^{2}(\Omega)\right)} \\
& \leq C\left(\left\|u_{0}\right\|_{L^{2}(\Omega)}+\|\mathbf{g}\|_{L^{2}\left((0, S) ; L^{2}(\Omega)\right)}\right) .
\end{aligned}
$$

Moreover, if $\mathbf{g} \in L^{\infty}\left((0, S) ; L^{2}(\Omega)\right)$, then $\mathbf{v}_{\boldsymbol{s}} \in L^{\infty}\left((0, S) ; H^{-1}(\Omega)\right)$, and

$$
\left\|\mathbf{v}_{s}\right\|_{L^{\infty}\left((0, S) ; H^{-1}(\Omega)\right.} \leq C\left(\left\|u_{0}\right\|_{L^{2}(\Omega)}+\|\mathbf{g}\|_{L^{2}\left((0, S) ; L^{2}(\Omega)\right)}+\|\mathbf{g}\|_{L^{\infty}\left((0, S) ; L^{2}(\Omega)\right)}\right) .
$$

Proof. See [8, Theorems 5 in $\S 7.1 .2]$ for proving (4.3). Next, we are going to prove that if $\mathbf{g} \in L^{\infty}\left((0, S) ; L^{2}(\Omega)\right)$, then $\mathbf{v}_{s} \in L^{\infty}\left((0, S) ; H^{-1}(\Omega)\right)$. From (1.4) $v_{s}=\operatorname{div} \nabla v+g$, from characterization of $H^{-1}(\Omega)$, see $(2.2)$ $\left\|\mathbf{v}_{s}(s)\right\|_{H^{-1}(\Omega)} \leq\|\mathbf{v}(s)\|_{H_{0}^{1}(\Omega)}+\|\mathbf{g}(s)\|_{L^{2}(\Omega)}$, therefore

$$
\left\|\mathbf{v}_{s}\right\|_{L^{\infty}\left((0, S) ; H^{-1}(\Omega)\right)} \leq\|\mathbf{v}\|_{L^{\infty}\left((0, S) ; H_{0}^{1}(\Omega)\right)}+\|\mathbf{g}\|_{L^{\infty}\left((0, S) ; L^{2}(\Omega)\right)} .
$$

Since (4.3), inequality (4.4) is accomplished, ending the proof.

\section{Proof of Theorems 3 and 4 (case $\beta<1)$}

This section collects several results concerning the regularity of a weak solution of (1.1), and further prove Theorems 3 and 4. All throughout this section, we 
assume that $\beta<1$ is fixed. Let $\mathbf{v}$ be the unique weak solution of (1.4), see Theorem 9. Define

$$
\mathbf{u}(t):=\mathbf{v}\left(\frac{1}{1-\beta} t^{1-\beta}\right),
$$

then it is easy to see that $\mathbf{u}$ is a weak solution of the parabolic problem (1.1). Which is not so clear at this moment, is the regularity attained by $\mathbf{u}$.

To unveil this question, we proceed with three technical Lemmata. The first Lemma precises the conditions on $\mathbf{f}$ ensuring that $\mathbf{g} \in L^{2}\left((0, S) ; L^{2}(\Omega)\right)$, to be able to apply the standard theory for parabolic problems, Theorems 9 and 10. The second Lemma relates the regularity of $\mathbf{u}$ with the regularity of $\mathbf{v}$, more specifically, states upper bounds of norms of $\mathbf{u}$ in terms of norms of $\mathbf{v}$. The third Lemma goes further in the regularity of $\mathbf{u}$, and as in the standard parabolic problem, we improve the regularity of a weak solution.

Lemma 1. Assume that $\mathbf{f} \in L^{\infty}\left((0, T) ; L^{2}(\Omega)\right)$. Let $\mathbf{g}$ be defined by

$$
\mathbf{g}(s):=\mathbf{f}\left([(1-\beta) s]^{\frac{1}{1-\beta}}\right),
$$

(see (1.5)), and $S$ defined by (1.3). Then, $\mathbf{g} \in L^{2}\left((0, S) ; L^{2}(\Omega)\right) \cap L^{\infty}((0, S)$; $\left.L^{2}(\Omega)\right)$, moreover

$$
\begin{aligned}
&\|\mathbf{g}\|_{L^{\infty}\left((0, S) ; L^{2}(\Omega)\right)}=\|\mathbf{f}\|_{L^{\infty}\left((0, T) ; L^{2}(\Omega)\right)} \\
&\|\mathbf{g}\|_{L^{2}\left((0, S) ; L^{2}(\Omega)\right)} \leq\left(\frac{T^{1-\beta}}{1-\beta}\right)^{1 / 2}\|\mathbf{f}\|_{L^{\infty}\left((0, T) ; L^{2}(\Omega)\right)} .
\end{aligned}
$$

Proof. By definition of $\mathbf{g}$, see (5.2), $\|\mathbf{g}(s)\|_{L^{2}(\Omega)}^{2}=\left\|\mathbf{f}\left([(1-\beta) s]^{\frac{1}{1-\beta}}\right)\right\|_{L^{2}(\Omega)}^{2}$. By definition of $\|\cdot\|_{L^{\infty}\left((0, S) ; L^{2}(\Omega)\right)}$, see (2.6), equality (5.3) is achieved.

By definition of $\|\cdot\|_{L^{2}\left((0, S) ; L^{2}(\Omega)\right)}$, see $(2.5)$, and of $s$, see (1.2),

$$
\|\mathbf{g}\|_{L^{2}\left((0, S) ; L^{2}(\Omega)\right)}^{2}=\int_{0}^{T} t^{-\beta}\|\mathbf{f}(t)\|_{L^{2}(\Omega)}^{2} d t \leq \frac{T^{1-\beta}}{1-\beta}\|\mathbf{f}\|_{L^{\infty}\left((0, T) ; L^{2}(\Omega)\right)}^{2},
$$

ending the proof.

Lemma 2. Assume that $\mathbf{f} \in L^{\infty}\left((0, T) ; L^{2}(\Omega)\right)$, and $u_{0} \in L^{2}(\Omega)$. Let $\mathbf{g}$ be defined by (5.2), and $S$ defined by (1.3). Let $\mathbf{v}$ be the unique weak solution of (1.4) provided by Theorem 9. Let $\mathbf{u}$ be given by (5.1). Then,

(i) $\mathbf{u} \in L^{2}\left((0, T) ; H_{0}^{1}(\Omega)\right)$ and $\|\mathbf{u}\|_{L^{2}\left((0, T) ; H_{0}^{1}(\Omega)\right)} \leq T^{\beta / 2}\|\mathbf{v}\|_{L^{2}\left((0, S) ; H_{0}^{1}(\Omega)\right)}$.

(ii) $\mathbf{u} \in C\left([0, T] ; L^{2}(\Omega)\right)$ and $\max _{t \in[0, T]}\|\mathbf{u}(t)\|_{L^{2}(\Omega)}=\max _{s \in[0, S]}\|\mathbf{v}(s)\|_{L^{2}(\Omega)}$.

(iii) $t^{\beta} \mathbf{u}_{t} \in L^{2}\left((0, T) ; H^{-1}(\Omega)\right), \mathbf{u}_{t} \in L^{1}\left((0, T) ; H^{-1}(\Omega)\right)$, and

$$
\left\|\mathbf{u}_{t}\right\|_{L^{1}\left((0, T) ; H^{-1}(\Omega)\right)}=\left\|\mathbf{v}_{s}\right\|_{L^{1}\left((0, S) ; H^{-1}(\Omega)\right)} .
$$

(iv) If $\beta<1 / 2$, then $\mathbf{u}_{t} \in L^{2}\left((0, T) ; H^{-1}(\Omega)\right)$, and

$$
\left\|\mathbf{u}_{t}\right\|_{L^{2}\left((0, T) ; H^{-1}(\Omega)\right)} \leq\left(\frac{T^{1-2 \beta}}{1-2 \beta}\right)^{1 / 2}\left\|\mathbf{v}_{s}\right\|_{L^{\infty}\left((0, S) ; H^{-1}(\Omega)\right)} .
$$


Proof. (i) By definition of $\mathbf{u}$, see (5.1), their norm in $H_{0}^{1}(\Omega)$ satisfy the following equality $\|\mathbf{u}(t)\|_{H_{0}^{1}(\Omega)}^{2}=\left\|\mathbf{v}\left(\frac{1}{1-\beta} t^{1-\beta}\right)\right\|_{H_{0}^{1}(\Omega)}^{2}$. By definition of the norm $\|\cdot\|_{L^{2}\left((0, T) ; H_{0}^{1}(\Omega)\right)}$, see $(2.5)$, and of $s$, see $(1.2)$,

$$
\begin{aligned}
& \|\mathbf{u}\|_{L^{2}\left((0, T) ; H_{0}^{1}(\Omega)\right)}^{2}=\int_{0}^{S}[(1-\beta) s]^{\frac{\beta}{1-\beta}}\|\mathbf{v}(s)\|_{H_{0}^{1}(\Omega)}^{2} d s \\
& \quad \leq[(1-\beta) S]^{\frac{\beta}{1-\beta}}\|\mathbf{v}\|_{L^{2}\left(0, S ; H_{0}^{1}(\Omega)\right)}^{2}=T^{\beta}\|\mathbf{v}\|_{L^{2}\left(0, S ; H_{0}^{1}(\Omega)\right)}^{2},
\end{aligned}
$$

ending part (i) of the proof.

(ii) Obviously $\|\mathbf{u}(t)\|_{L^{2}(\Omega)}^{2}=\left\|\mathbf{v}\left(\frac{1}{1-\beta} t^{1-\beta}\right)\right\|_{L^{2}(\Omega)}^{2}$. Moreover, when $t \in$ $[0, T]$, then $s \in[0, S]$, and so part (ii) is achieved.

(iii) From definition of $\mathbf{u}$, see (5.1)

$$
\mathbf{u}_{t}(t)=t^{-\beta} \mathbf{v}_{s}\left(\frac{1}{1-\beta} t^{1-\beta}\right)
$$

therefore $t^{\beta} \mathbf{u}_{t} \in L^{2}\left((0, T) ; H^{-1}(\Omega)\right)$. Moreover

$$
\begin{gathered}
\left\|\mathbf{u}_{t}\right\|_{L^{1}\left((0, T) ; H^{-1}(\Omega)\right)}=\int_{0}^{T} t^{-\beta}\left\|\mathbf{v}_{s}\left(\frac{1}{1-\beta} t^{1-\beta}\right)\right\|_{H^{-1}(\Omega)} d t \\
=\int_{0}^{S}\left\|\mathbf{v}_{s}(s)\right\|_{H^{-1}(\Omega)} d s=\left\|\mathbf{v}_{s}\right\|_{L^{1}\left((0, S) ; H^{-1}(\Omega)\right)} .
\end{gathered}
$$

(iv) From definition, and (5.4), if $\beta<1 / 2$,

$$
\begin{aligned}
& \left\|\mathbf{u}_{t}\right\|_{L^{2}\left((0, T) ; H^{-1}(\Omega)\right)}^{2}=\int_{0}^{T} t^{-2 \beta}\left\|\mathbf{v}_{s}\left(\frac{1}{1-\beta} t^{1-\beta}\right)\right\|_{H^{-1}(\Omega)}^{2} d t=\int_{0}^{S}[(1-\beta) s]^{\frac{-\beta}{1-\beta}} \\
& \quad \times\left\|\mathbf{v}_{s}(s)\right\|_{H^{-1}(\Omega)}^{2} d s \leq\left\|\mathbf{v}_{s}\right\|_{L^{\infty}\left((0, S) ; H^{-1}(\Omega)\right)}^{2} \frac{1}{1-2 \beta}[(1-\beta) S]^{\frac{1-2 \beta}{1-\beta}}
\end{aligned}
$$

which ends the proof.

Lemma 3. Assume that $\mathbf{f} \in L^{\infty}\left((0, T) ; L^{2}(\Omega)\right)$, and $u_{0} \in L^{2}(\Omega)$ are given. Let $\mathbf{g}$ be defined by (5.2), and $S$ defined by (1.3). Let $\mathbf{v}$ be the unique weak solution of (1.4) provided by Theorem 9. Let $\mathbf{u}$ be given by (5.1). Then,

(i) $\mathbf{u} \in L^{2}\left((0, T) ; H^{2}(\Omega)\right)$ and $\|\mathbf{u}\|_{L^{2}\left((0, T) ; H^{2}(\Omega)\right)} \leq T^{\beta / 2}\|\mathbf{v}\|_{L^{2}\left((0, S) ; H^{2}(\Omega)\right)}$.

(ii) $\mathbf{u} \in C\left([0, T] ; H_{0}^{1}(\Omega)\right)$ and $\max _{t \in[0, T]}\|\mathbf{u}(t)\|_{H_{0}^{1}(\Omega)}=\max _{s \in[0, S]}\|\mathbf{v}(s)\|_{H_{0}^{1}(\Omega)}$.

(iii) $\mathbf{u}_{t} \in L^{1}\left((0, T) ; L^{2}(\Omega)\right)$ and $\left\|\mathbf{u}_{t}\right\|_{L^{1}\left((0, T) ; L^{2}(\Omega)\right)}=\left\|\mathbf{v}_{s}\right\|_{L^{1}\left((0, S) ; L^{2}(\Omega)\right)}$.

Proof. (i) By definition of $\mathbf{u}$, see (5.1), $\|\mathbf{u}(t)\|_{H^{2}(\Omega)}^{2}=\left\|\mathbf{v}\left(\frac{1}{1-\beta} t^{1-\beta}\right)\right\|_{H^{2}(\Omega)}^{2}$. By definition of $\|\cdot\|_{L^{2}\left((0, T) ; H^{2}(\Omega)\right)}$, see (2.5), and of $s$, see (1.2),

$$
\begin{aligned}
& \|\mathbf{u}\|_{L^{2}\left((0, T) ; H^{2}(\Omega)\right)}^{2}=\int_{0}^{S}[(1-\beta) s]^{\frac{\beta}{1-\beta}}\|\mathbf{v}(s)\|_{H^{2}(\Omega)}^{2} d s \\
& \quad \leq[(1-\beta) S]^{\frac{\beta}{1-\beta}}\|\mathbf{v}\|_{L^{2}\left((0, S) ; H^{2}(\Omega)\right)}^{2}=T^{\beta}\|\mathbf{v}\|_{L^{2}\left((0, S) ; H^{2}(\Omega)\right)}^{2},
\end{aligned}
$$


ending part (i) of the proof.

(ii) Obviously $\|\mathbf{u}(t)\|_{H_{0}^{1}(\Omega)}^{2}=\left\|\mathbf{v}\left(\frac{1}{1-\beta} t^{1-\beta}\right)\right\|_{H_{0}^{1}(\Omega)}^{2}$. Moreover, when $t \in$ $[0, T]$, then $s \in[0, S]$, and so part (ii) of the proof is achieved.

(iii) From (5.4) $\left\|\mathbf{u}_{t}(t)\right\|_{L^{2}(\Omega)}=t^{-\beta}\left\|\mathbf{v}_{s}\left(\frac{1}{1-\beta} t^{1-\beta}\right)\right\|_{L^{2}(\Omega)}$, and

$$
\begin{gathered}
\left\|\mathbf{u}_{t}\right\|_{L^{1}\left((0, T) ; L^{2}(\Omega)\right)}=\int_{0}^{T} t^{-\beta}\left\|\mathbf{v}_{s}\left(\frac{1}{1-\beta} t^{1-\beta}\right)\right\|_{L^{2}(\Omega)} d t \\
=\int_{0}^{S}\left\|\mathbf{v}_{s}(s)\right\|_{L^{2}(\Omega)} d s=\left\|\mathbf{v}_{s}\right\|_{L^{1}\left((0, S) ; L^{2}(\Omega)\right)}^{2},
\end{gathered}
$$

ending the proof.

Proof. [Proof of Theorem 3] It is a consequence of Theorem 9, Lemma 1 and Lemma 2.

Proof. [Proof of Theorem 4] It is a consequence of Theorem 10, Lemma 1 and Lemma 3.

\section{Proof of Theorems 5 and $6\left(\right.$ case $\left.\beta=1, t_{1}>0\right)$}

This section collects several results concerning the regularity of a weak solution of $(1.7)_{t_{1}}$, and further prove Theorems 5 and 6 . All throughout this section, we assume that $\beta=1$ and $t_{1}>0$ is fixed.

Given $\mathbf{f}$, let us define

$$
\mathbf{g}(\theta):=\mathbf{f}\left(t_{1} e^{\theta}\right)
$$

see (1.11). Let $\mathbf{v}$ be the unique weak solution of (1.10), given by the formula of variation of constants,

$$
\mathbf{v}(\theta)=\sum_{i=1}^{\infty} e^{-\lambda_{i} \theta}\left\langle u_{1}, \varphi_{i}\right\rangle \varphi_{i}+\sum_{i=1}^{\infty}\left(\int_{0}^{\theta} e^{-\lambda_{i}(\theta-\sigma)}\left\langle\mathbf{g}(\sigma), \varphi_{i}\right\rangle d \sigma\right) \varphi_{i}
$$

(see $[5,8,13,22,25]$, see also Theorem 9 and equation (4.1)).

Define

$$
\mathbf{u}(t):=\mathbf{v}\left(\ln \left(t / t_{1}\right)\right),
$$

then it is easy to see that $\mathbf{u}$ is a weak solution of the parabolic problem $(1.7)_{t_{1}}$.

To study their regularity, we proceed with three technical Lemmata. The first Lemma precise the conditions on $\mathbf{f}$ ensuring that $\mathbf{g} \in L^{2}\left((0, \Theta) ; L^{2}(\Omega)\right)$, to be able to apply the standard theory for parabolic problems, Theorems 9 and 10. The second and third Lemmata relates the regularity of $\mathbf{u}$ with the regularity of $\mathbf{v}$, stating upper bounds of norms of $\mathbf{u}$ in terms of norms of $\mathbf{v}$.

Lemma 4. Assume that $\mathbf{f} \in L^{\infty}\left((0, T) ; L^{2}(\Omega)\right)$. Let $\mathbf{g}$ be defined by (6.1), and $\Theta$ be defined by (1.9). Let us fix an arbitrary $t_{1} \in(0, T)$. Then, $\mathbf{g} \in$ $L^{2}\left((0, \Theta) ; L^{2}(\Omega)\right) \cap L^{\infty}\left((0, T) ; L^{2}(\Omega)\right)$, moreover

$$
\begin{aligned}
& \|\mathbf{g}\|_{L^{\infty}\left((0, \Theta) ; L^{2}(\Omega)\right)} \leq\|\mathbf{f}\|_{L^{\infty}\left((0, T) ; L^{2}(\Omega)\right)}, \\
& \|\mathbf{g}\|_{L^{2}\left((0, \Theta) ; L^{2}(\Omega)\right)} \leq\left[\ln \left(T / t_{1}\right)\right]^{1 / 2}\|\mathbf{f}\|_{L^{\infty}\left((0, T) ; L^{2}(\Omega)\right)} .
\end{aligned}
$$


Furthermore, if there exists a constant $C>0$ (depending on $T$ ), such that

$$
\int_{0}^{T} \frac{1}{t}\|\mathbf{f}(t)\|_{L^{2}(\Omega)}^{2} d t \leq C
$$

then

$$
\|\mathbf{g}\|_{L^{2}\left((0, \Theta) ; L^{2}(\Omega)\right)} \leq\left(\int_{0}^{T} \frac{1}{t}|\mathbf{f}(t)|_{L^{2}(\Omega)}^{2} d t\right)^{1 / 2}
$$

Proof. By definition of $\mathbf{g}$, see (6.1), $\|\mathbf{g}(\theta)\|_{L^{2}(\Omega)}^{2}=\left\|\mathbf{f}\left(t_{1} e^{\theta}\right)\right\|_{L^{2}(\Omega)}^{2}$. By definition of $\|\cdot\|_{L^{\infty}\left((0, \Theta) ; L^{2}(\Omega)\right)}$, see $(2.6),\|\mathbf{g}\|_{L^{\infty}\left((0, \Theta) ; L^{2}(\Omega)\right)}=\|\mathbf{f}\|_{L^{\infty}\left(\left(t_{1}, T\right) ; L^{2}(\Omega)\right)} \leq$ $\|\mathbf{f}\|_{L^{\infty}\left((0, T) ; L^{2}(\Omega)\right)}$. By definition of $\|\cdot\|_{L^{2}\left((0, \Theta) ; L^{2}(\Omega)\right)}$, see $(2.5)$, and of $\theta$, see $(1.8)$,

$$
\|\mathbf{g}\|_{L^{2}\left((0, \Theta) ; L^{2}(\Omega)\right)}^{2}=\int_{t_{1}}^{T} \frac{1}{t}\|\mathbf{f}(t)\|_{L^{2}(\Omega)}^{2} d t \leq \ln \left(\frac{T}{t_{1}}\right)\|\mathbf{f}\|_{L^{\infty}\left((0, T) ; L^{2}(\Omega)\right)}^{2} .
$$

Moreover, $\|\mathbf{g}\|_{L^{2}\left((0, \Theta) ; L^{2}(\Omega)\right)}^{2}=\int_{t_{1}}^{T} \frac{1}{t}\|\mathbf{f}(t)\|_{L^{2}(\Omega)}^{2} d t \leq \int_{0}^{T} \frac{1}{t}\|\mathbf{f}(t)\|_{L^{2}(\Omega)}^{2} d t$, ending the proof.

Lemma 5. Assume that $\mathbf{f} \in L^{\infty}\left((0, T) ; L^{2}(\Omega)\right)$, assume also that $u_{1} \in L^{2}(\Omega)$. Let $\mathbf{g}$ be defined by (6.1). Let $\mathbf{v}$ be the unique weak solution of (1.10) provided by Theorem 9. Let $\mathbf{u}$ be given by (6.2). Then,

(i) $\mathbf{u} \in L^{2}\left(\left(t_{1}, T\right) ; H_{0}^{1}(\Omega)\right),\|\mathbf{u}\|_{L^{2}\left(\left(t_{1}, T\right) ; H_{0}^{1}(\Omega)\right)} \leq T^{1 / 2}\|\mathbf{v}\|_{L^{2}\left((0, \Theta) ; H_{0}^{1}(\Omega)\right)}$.

(ii) $\mathbf{u} \in C\left(\left[t_{1}, T\right] ; L^{2}(\Omega)\right)$, and $\max _{t \in\left[t_{1}, T\right]}\|\mathbf{u}(t)\|_{L^{2}(\Omega)}=\max _{\theta \in[0, \Theta]}\|\mathbf{v}(\theta)\|_{L^{2}(\Omega)}$.

(iii) $\mathbf{u}_{t} \in L^{1}\left(\left(t_{1}, T\right) ; H^{-1}(\Omega)\right),\left\|\mathbf{u}_{t}\right\|_{L^{1}\left(\left(t_{1}, T\right) ; H^{-1}(\Omega)\right)}=\left\|\mathbf{v}_{s}\right\|_{L^{1}\left((0, \Theta) ; H^{-1}(\Omega)\right)}$.

Proof. (i) By definition of $\mathbf{u}$, see (6.2), their $H_{0}^{1}(\Omega)$-norm satisfy the following $\|\mathbf{u}(t)\|_{H_{0}^{1}(\Omega)}^{2}=\left\|\mathbf{v}\left(\ln \left(t / t_{1}\right)\right)\right\|_{H_{0}^{1}(\Omega)}^{2}$. By definition of $\|\cdot\|_{L^{2}\left(\left(t_{1}, T\right) ; H_{0}^{1}(\Omega)\right)^{-n o r m},}$ see (2.5), and of $\theta$, see (1.8),

$$
\begin{gathered}
\|\mathbf{u}\|_{L^{2}\left(\left(t_{1}, T\right) ; H_{0}^{1}(\Omega)\right)}^{2}=\int_{t_{1}}^{T}\left\|\mathbf{v}\left(\ln \left(\frac{t}{t_{1}}\right)\right)\right\|_{H_{0}^{1}(\Omega)}^{2} d t=\int_{0}^{\Theta} t_{1} e^{\theta}\|\mathbf{v}(\theta)\|_{H_{0}^{1}(\Omega)}^{2} d \theta \\
\leq t_{1} e^{\Theta}\|\mathbf{v}\|_{L^{2}\left((0, \Theta) ; H_{0}^{1}(\Omega)\right)}^{2}=T\|\mathbf{v}\|_{L^{2}\left((0, \Theta) ; H_{0}^{1}(\Omega)\right)}^{2}
\end{gathered}
$$

ending part (i) of the proof.

(ii) Obviously $\|\mathbf{u}(t)\|_{L^{2}(\Omega)}^{2}=\left\|\mathbf{v}\left(\ln \left(\frac{t}{t_{1}}\right)\right)\right\|_{L^{2}(\Omega)}^{2}$. Moreover, when $t \in\left[t_{1}, T\right]$, then $\theta \in[0, \Theta]$, and so part (ii) of the proof is achieved.

(iii) From definition of $\mathbf{u}$, see $(6.2)$

$$
\mathbf{u}_{t}(t)=\frac{1}{t} \mathbf{v}_{\theta}\left(\ln \left(t / t_{1}\right)\right)
$$


therefore $\left\|\mathbf{u}_{t}(t)\right\|_{H^{-1}(\Omega)}=\frac{1}{t}\left\|\mathbf{v}_{\theta}\left(\ln \left(t / t_{1}\right)\right)\right\|_{H^{-1}(\Omega)}$, and

$$
\left\|\mathbf{u}_{t}\right\|_{L^{1}\left(\left(t_{1}, T\right) ; H^{-1}(\Omega)\right)}=\int_{0}^{\Theta}\left\|\mathbf{v}_{\theta}(\theta)\right\|_{H^{-1}(\Omega)} d s=\left\|\mathbf{v}_{s}\right\|_{L^{1}\left((0, \Theta) ; H^{-1}(\Omega)\right)},
$$

which ends the proof.

Lemma 6. Assume that $\mathbf{f} \in L^{\infty}\left((0, T) ; L^{2}(\Omega)\right)$, and $u_{1} \in L^{2}(\Omega)$. Let $\mathbf{g}$ be defined by (6.1). Let $\mathbf{v}$ be the unique weak solution of (1.10) provided by Theorem 9. Let $\mathbf{u}$ be given by (6.2). Then,

(i) $\mathbf{u} \in L^{2}\left(\left(t_{1}, T\right) ; H^{2}(\Omega)\right),\|\mathbf{u}\|_{L^{2}\left(\left(t_{1}, T\right) ; H^{2}(\Omega)\right)} \leq T^{1 / 2}\|\mathbf{v}\|_{L^{2}\left((0, \Theta) ; H^{2}(\Omega)\right)}$.

(ii) $\mathbf{u} \in C\left(\left[t_{1}, T\right] ; H_{0}^{1}(\Omega)\right)$, and $\max _{t \in\left[t_{1}, T\right]}\|\mathbf{u}(t)\|_{H_{0}^{1}(\Omega)}=\max _{\theta \in[0, \Theta]}\|\mathbf{v}(\theta)\|_{H_{0}^{1}(\Omega)}$.

(iii) $\mathbf{u}_{t} \in L^{1}\left(\left(t_{1}, T\right) ; L^{2}(\Omega)\right)$, and $\left\|\mathbf{u}_{t}\right\|_{L^{1}\left(\left(t_{1}, T\right) ; L^{2}(\Omega)\right)}=\left\|\mathbf{v}_{s}\right\|_{L^{1}\left((0, \Theta) ; L^{2}(\Omega)\right)}$.

Proof. (i) By definition of $\mathbf{u}$, see $(6.2),\|\mathbf{u}(t)\|_{H^{2}(\Omega)}^{2}=\left\|\mathbf{v}\left(\ln \left(t / t_{1}\right)\right)\right\|_{H^{2}(\Omega)}^{2}$. By definition of $\|\cdot\|_{L^{2}\left(\left(t_{1}, T\right) ; H^{2}(\Omega)\right)}$, see (2.5), and of $\theta$, see (1.8),

$$
\begin{aligned}
& \|\mathbf{u}\|_{L^{2}\left(t_{1}, T ; H^{2}(\Omega)\right)}^{2}=\int_{0}^{\Theta} t_{1} e^{\theta}\|\mathbf{v}(\theta)\|_{H^{2}(\Omega)}^{2} d \theta \\
& \leq t_{1} e^{\Theta}\|\mathbf{v}\|_{L^{2}\left((0, \Theta) ; H^{2}(\Omega)\right)}^{2}=T\|\mathbf{v}\|_{L^{2}\left((0, \Theta) ; H^{2}(\Omega)\right)}^{2},
\end{aligned}
$$

ending part (i) of the proof.

(ii) Obviously $\|\mathbf{u}(t)\|_{H_{0}^{1}(\Omega)}^{2}=\left\|\mathbf{v}\left(\ln \left(\frac{t}{t_{1}}\right)\right)\right\|_{H_{0}^{1}(\Omega)}^{2}$. Moreover, when $t \in\left[t_{1}, T\right]$, then $\theta \in[0, \Theta]$, and so part (ii) of the proof is achieved.

(iii) From (6.3) $\left\|\mathbf{u}_{t}(t)\right\|_{L^{2}(\Omega)}=\frac{1}{t}\left\|\mathbf{v}_{\theta}\left(\ln \left(t / t_{1}\right)\right)\right\|_{L^{2}(\Omega)}$, and

$$
\left\|\mathbf{u}_{t}\right\|_{L^{1}\left(\left(t_{1}, T\right) ; L^{2}(\Omega)\right)}=\int_{0}^{\Theta}\left\|\mathbf{v}_{\theta}(\theta)\right\|_{L^{2}(\Omega)} d \theta=\left\|\mathbf{v}_{\theta}\right\|_{L^{1}\left((0, \Theta) ; L^{2}(\Omega)\right)}^{2},
$$

ending the proof.

Proof. [Proof of Theorems 5 and 6.] We merge both proofs due to their similarity. Fixing an arbitrary $t_{1}>0$, and $u_{1} \in L^{2}(\Omega)$, the existence of a unique solution of $(1.7)_{t_{1}}$ for $t \in\left(t_{1}, T\right)$ is a consequence of Theorem 9 , Lemma 4 and Lemma 5. Moreover, its regularity is a consequence of Theorem 10, Lemma 4 and Lemma 6.

\section{Proof of Theorems 7 and $8\left(\right.$ case $\left.\beta=1, t_{1} \rightarrow 0\right)$}

This section collects several results concerning the regularity of a weak solution of (1.15), and further prove Theorems 7 and 8. All throughout this section, we assume that $\beta=1$. 
Proof. [Proof of Theorems 7 and 8.] We also merge both proofs thanks to their similarity. Let us denote by $u\left(t ; t_{1}, u_{1}\right)$ the unique solution of $(1.7)_{t_{1}}$ for $t \in\left(t_{1}, T\right)$ provided by Theorems 5 and 6 , and defined by (1.12).

In part I), fixing any $t>0$, we analyze the asymptotic behavior of $u\left(t ; t_{1}, u_{1}\right)$ as $t_{1} \rightarrow 0$, and see that, for any $u_{1} \in L^{2}(\Omega)$, it is independent of $u_{1}$. Let us denote it by $\mathbf{u}(t)$. In part II) we analyze the regularity of $\mathbf{u}(t)$, and prove that $\mathbf{u} \in L^{2}\left((0, T), H^{2}(\Omega)\right)$ and that $t \mathbf{u}_{t} \in L^{2}\left((0, T), L^{2}(\Omega)\right)$. In part III) we prove that $\mathbf{u} \in C\left((0, T], L^{2}(\Omega)\right)$. Finally in part IV), we study the asymptotic behavior of $\mathbf{u}(t)$ as $t \rightarrow 0$.

I) Let $u(t)$ be the solution of $(1.7)_{t_{1}}$ on $\left(t_{1}, T\right)$ given by (1.12). Fixing any $t>0$, let us define $\mathbf{u}$ by $(1.13)$. We next see that $\mathbf{u}(t):=\lim _{t_{1} \rightarrow 0} u\left(t ; t_{1}, u_{1}\right)$.

Let us first look at the first term on the right hand side in the equation (1.12). From Parseval's identity and since $\lambda_{1}<\lambda_{2} \leq \cdots \leq \lambda_{i} \leq \cdots$, fixing any $t>0$, for any $t_{1}<t$ we can write

$$
\begin{gathered}
\left\|\sum_{i=1}^{\infty}\left(\frac{t_{1}}{t}\right)^{\lambda_{i}}\left\langle u_{1}, \varphi_{i}\right\rangle \varphi_{i}\right\|_{L^{2}(\Omega)}=\left(\frac{t_{1}}{t}\right)^{\lambda_{1}}\left[\sum_{i=1}^{\infty}\left(\frac{t_{1}}{t}\right)^{2\left(\lambda_{i}-\lambda_{1}\right)}\left|\left\langle u_{1}, \varphi_{i}\right\rangle\right|^{2}\right]^{1 / 2} \\
\leq\left(t_{1} / t\right)^{\lambda_{1}}\left\|u_{1}\right\|_{L^{2}(\Omega)} \rightarrow 0 \quad \text { as } \quad t_{1} \rightarrow 0 .
\end{gathered}
$$

Now we substract the limit of $u$ as $t_{1} \rightarrow 0$ given by (1.13) minus the second term in the right hand side of (1.12) to obtain

$$
\begin{gathered}
\sum_{i=1}^{\infty} t^{-\lambda_{i}}\left(\int_{0}^{t} \sigma^{\lambda_{i}-1}\left\langle\mathbf{f}(\sigma), \varphi_{i}\right\rangle d \sigma-\int_{t_{1}}^{t} \sigma^{\lambda_{i}-1}\left\langle\mathbf{f}(\sigma), \varphi_{i}\right\rangle d \sigma\right) \varphi_{i} \\
=\sum_{i=1}^{\infty} t^{-\lambda_{i}}\left(\int_{0}^{t_{1}} \sigma^{\lambda_{i}-1}\left\langle\mathbf{f}(\sigma), \varphi_{i}\right\rangle d \sigma\right) \varphi_{i}
\end{gathered}
$$

Assuming that $\mathbf{f} \in \mathbf{X} \cap C\left([0, T] ; L^{2}(\Omega)\right)$ (see (3.3)-(3.4)),

$$
\begin{aligned}
& \left\|\sum_{i=1}^{\infty} t^{-\lambda_{i}}\left(\int_{0}^{t_{1}} \sigma^{\lambda_{i}-1}\left\langle\mathbf{f}(\sigma), \varphi_{i}\right\rangle d \sigma\right) \varphi_{i}\right\|_{H^{2}(\Omega)}^{2}=\sum_{i=1}^{\infty} \lambda_{i}^{2} t^{-2 \lambda_{i}} \\
& \quad \times\left(\int_{0}^{t_{1}} \sigma^{\lambda_{i}-1}\left\langle\mathbf{f}(\sigma), \varphi_{i}\right\rangle d \sigma\right)^{2} \leq \sum_{i=1}^{\infty} \lambda_{i}^{2} t^{-2 \lambda_{i}} \sup _{\sigma \in\left[0, t_{1}\right]}\left|\left\langle\mathbf{f}(\sigma), \varphi_{i}\right\rangle\right|^{2} \\
& \quad \times\left(\int_{0}^{t_{1}} \sigma^{\lambda_{i}-1} d \sigma\right)^{2}=\sum_{i=1}^{\infty}\left(\frac{t_{1}}{t}\right)^{2 \lambda_{i}} \sup _{\sigma \in\left[0, t_{1}\right]}\left|\left\langle\mathbf{f}(\sigma), \varphi_{i}\right\rangle\right|^{2} \\
& =\left(\frac{t_{1}}{t}\right)^{2 \lambda_{1}} \sum_{i=1}^{\infty}\left(\frac{t_{1}}{t}\right)^{2\left(\lambda_{i}-\lambda_{1}\right)} \sup _{\sigma \in\left[0, t_{1}\right]}\left|\left\langle\mathbf{f}(\sigma), \varphi_{i}\right\rangle\right|^{2} \\
& \leq\left(\frac{t_{1}}{t}\right)^{2 \lambda_{1}} \sum_{i=1}^{\infty} \sup _{\sigma \in\left[0, t_{1}\right]}\left|\left\langle\mathbf{f}(\sigma), \varphi_{i}\right\rangle\right|^{2} .
\end{aligned}
$$

Since hypothesis (3.5) on $\mathbf{f}$,

$$
\left\|\mathbf{u}(t)-u\left(t ; t_{1}, u_{1}\right)\right\|_{L^{2}(\Omega)}^{2} \leq\left(\frac{t_{1}}{t}\right)^{2 \lambda_{1}}\left(\left\|u_{1}\right\|_{L^{2}(\Omega)}+\sum_{i=1}^{\infty} \sup _{\sigma \in\left[0, t_{1}\right]}\left|\left\langle\mathbf{f}(\sigma), \varphi_{i}\right\rangle\right|^{2}\right) \rightarrow 0
$$


as $t_{1} \rightarrow 0$, and $\lim _{t_{1} \rightarrow 0} u\left(t ; t_{1}, u_{1}\right)=\mathbf{u}(t)$ in $L^{2}(\Omega)$.

II) At least formally

$$
-\Delta \mathbf{u}(t)=\sum_{i=1}^{\infty} \lambda_{i} t^{-\lambda_{i}}\left(\int_{0}^{t} \sigma^{\lambda_{i}-1}\left\langle\mathbf{f}(\sigma), \varphi_{i}\right\rangle d \sigma\right) \varphi_{i}
$$

and reasoning as above, for any $t>0$

$$
\begin{gathered}
\|\mathbf{u}(t)\|_{H^{2}(\Omega)}^{2}=\sum_{i=1}^{\infty} \lambda_{i}^{2} t^{-2 \lambda_{i}}\left(\int_{0}^{t} \sigma^{\lambda_{i}-1}\left\langle\mathbf{f}(\sigma), \varphi_{i}\right\rangle d \sigma\right)^{2} \leq \sum_{i=1}^{\infty} \lambda_{i}^{2} t^{-2 \lambda_{i}} \\
\times \sup _{\sigma \in[0, t]}\left|\left\langle\mathbf{f}(\sigma), \varphi_{i}\right\rangle\right|^{2}\left(\int_{0}^{t} \sigma^{\lambda_{i}-1} d \sigma\right)^{2}=\sum_{i=1}^{\infty} \sup _{\sigma \in[0, t]}\left|\left\langle\mathbf{f}(\sigma), \varphi_{i}\right\rangle\right|^{2} .
\end{gathered}
$$

Since $\mathbf{f} \in \mathbf{X}($ see $(3.3)-(3.4))$, then $\left(\int_{0}^{T}\|\mathbf{u}(t)\|_{H^{2}(\Omega)}^{2} d t\right)^{1 / 2} \leq\|\mathbf{f}\|_{\mathbf{X}}^{2}$, and $\mathbf{u} \in$ $L^{2}\left((0, T), H^{2}(\Omega)\right)$. Also, for any $t>0$,

$$
\begin{aligned}
\mathbf{u}_{t}(t) & =\sum_{i=1}^{\infty}-\lambda_{i} t^{-\lambda_{i}-1}\left(\int_{0}^{t} \sigma^{\lambda_{i}-1}\left\langle\mathbf{f}(\sigma), \varphi_{i}\right\rangle d \sigma\right) \varphi_{i}+\sum_{i=1}^{\infty} t^{-1}\left\langle\mathbf{f}(t), \varphi_{i}\right\rangle \varphi_{i} \\
& =t^{-1} \sum_{i=1}^{\infty}\left[-\lambda_{i} t^{-\lambda_{i}}\left(\int_{0}^{t} \sigma^{\lambda_{i}}\left\langle\mathbf{f}(\sigma), \varphi_{i}\right\rangle d \sigma\right)+\left\langle\mathbf{f}(t), \varphi_{i}\right\rangle\right] \varphi_{i} \\
& =t^{-1} \sum_{i=1}^{\infty} \lambda_{i} t^{-\lambda_{i}}\left(\int_{0}^{t} \sigma^{\lambda_{i}}\left\langle\mathbf{f}(t)-\mathbf{f}(\sigma), \varphi_{i}\right\rangle d \sigma\right) \varphi_{i} .
\end{aligned}
$$

Moreover,

$$
\begin{aligned}
& \left(t\left\|\mathbf{u}_{t}(t)\right\|_{L^{2}(\Omega)}\right)^{2}=\sum_{i=1}^{\infty} \lambda_{i}^{2} t^{-2 \lambda_{i}}\left(\int_{0}^{t} \sigma^{\lambda_{i}-1}\left\langle\mathbf{f}(t)-\mathbf{f}(\sigma), \varphi_{i}\right\rangle d \sigma\right)^{2} \\
& \leq \sum_{i=1}^{\infty} \lambda_{i}^{2} t^{-2 \lambda_{i}} \sup _{\sigma \in[0, t]}\left|\left\langle\mathbf{f}(t)-\mathbf{f}(\sigma), \varphi_{i}\right\rangle\right|^{2}\left(\int_{0}^{t} \sigma^{\lambda_{i}-1} d \sigma\right)^{2} \\
& =\sum_{i=1}^{\infty} \sup _{\sigma \in[0, t]}\left|\left\langle\mathbf{f}(t)-\mathbf{f}(\sigma), \varphi_{i}\right\rangle\right|^{2}
\end{aligned}
$$

Since

$$
\begin{aligned}
& \sum_{i=1}^{\infty} \sup _{\sigma \in[0, t]}\left|\left\langle\mathbf{f}(t)-\mathbf{f}(\sigma), \varphi_{i}\right\rangle\right|^{2} \leq \sum_{i=1}^{\infty}\left(\left|\left\langle\mathbf{f}(t), \varphi_{i}\right\rangle\right|+\sup _{\sigma \in[0, t]}\left|\left\langle\mathbf{f}(\sigma), \varphi_{i}\right\rangle\right|\right)^{2}, \\
& (a+b)^{2} \leq 2\left(a^{2}+b^{2}\right), \quad \sum_{i=1}^{\infty}\left|\left\langle\mathbf{f}(t), \varphi_{i}\right\rangle\right|^{2}=\|\mathbf{f}(t)\|_{L^{2}(\Omega)}^{2},
\end{aligned}
$$

then

$$
\int_{0}^{T}\left(t\left\|\mathbf{u}_{t}(t)\right\|_{L^{2}(\Omega)}\right)^{2} d t \leq 2\|\mathbf{f}\|_{L^{2}\left((0, T)^{2}, L^{2}(\Omega)\right)}^{2}+2\|\mathbf{f}\|_{\mathbf{X}}^{2} \leq 4\|\mathbf{f}\|_{\mathbf{X}}^{2}
$$


and $t \mathbf{u}_{t} \in L^{2}\left((0, T), L^{2}(\Omega)\right)$.

III) Fix any $t>0$. From (1.13) we deduce

$$
\begin{aligned}
\mathbf{u}(t+h)-\mathbf{u}(t) & =\sum_{i=1}^{\infty}(t+h)^{-\lambda_{i}}\left(\int_{0}^{t+h}\left\langle\mathbf{f}(\sigma), \varphi_{i}\right\rangle \sigma^{\lambda_{i}-1} d \sigma\right) \varphi_{i} \\
& -\sum_{i=1}^{\infty} t^{-\lambda_{i}}\left(\int_{0}^{t}\left\langle\mathbf{f}(\sigma), \varphi_{i}\right\rangle \sigma^{\lambda_{i}-1} d \sigma\right) \varphi_{i}
\end{aligned}
$$

therefore, for $h>0$ we can write

$$
\begin{aligned}
& \|\mathbf{u}(t+h)-\mathbf{u}(t)\|_{L^{2}(\Omega)}^{2}=\sum_{i=1}^{\infty}\left[t^{-\lambda_{i}}\left(\int_{0}^{t}\left\langle\mathbf{f}(\sigma), \varphi_{i}\right\rangle \sigma^{\lambda_{i}-1} d \sigma\right)\left(\left(\frac{t+h}{t}\right)^{-\lambda_{i}}-1\right)\right. \\
& \left.+(t+h)^{-\lambda_{i}}\left(\int_{t}^{t+h}\left\langle\mathbf{f}(\sigma), \varphi_{i}\right\rangle \sigma^{\lambda_{i}-1} d \sigma\right)\right]^{2}=: \sum_{i=1}^{\infty}\left[A_{i}(t, h)+B_{i}(t, h)\right]^{2} .
\end{aligned}
$$

Observe that $\left(\frac{t+h}{t}\right)^{-\lambda_{i}}-1=\int_{0}^{1} \psi(\theta) d \theta, \quad$ with

$$
\psi(\theta):=\frac{d}{d \theta}\left[\left(\frac{t+h \theta}{t}\right)^{-\lambda_{i}}\right]=-\lambda_{i}\left(\frac{t+h \theta}{t}\right)^{-\lambda_{i}-1} \frac{h}{t} .
$$

Then

$$
\begin{aligned}
& \left|\left(\frac{t+h}{t}\right)^{-\lambda_{i}}-1\right| \leq \lambda_{i} \frac{h}{t} \int_{0}^{1}\left(\frac{t}{t+h \theta}\right)^{\lambda_{i}+1} d \theta \leq \lambda_{i} \frac{h}{t} \\
& \left|A_{i}(t, h)\right| \leq h \lambda_{i} t^{-\lambda_{i}-1}\left(\int_{0}^{t}\left|\left\langle\mathbf{f}(\sigma), \varphi_{i}\right\rangle\right| \sigma^{\lambda_{i}-1} d \sigma\right) \leq \frac{h}{t} \sup _{\sigma \in[0, t]}\left|\left\langle\mathbf{f}(\sigma), \varphi_{i}\right\rangle\right| .
\end{aligned}
$$

On the other side

$$
\begin{aligned}
& \left|B_{i}(t, h)\right| \leq(t+h)^{-\lambda_{i}} \sup _{\sigma \in[t, t+h]}\left|\left\langle\mathbf{f}(\sigma), \varphi_{i}\right\rangle\right|\left(\frac{(t+h)^{\lambda_{i}}-t^{\lambda_{i}}}{\lambda_{i}}\right) \\
& \quad=\frac{1}{\lambda_{i}} \sup _{\sigma \in[t, t+h]}\left|\left\langle\mathbf{f}(\sigma), \varphi_{i}\right\rangle\right|\left(1-\left(\frac{t}{(t+h)}\right)^{\lambda_{i}}\right) \leq \frac{h}{t} \sup _{\sigma \in[t, t+h]}\left|\left\langle\mathbf{f}(\sigma), \varphi_{i}\right\rangle\right| .
\end{aligned}
$$

Consequently

$$
\|\mathbf{u}(t+h)-\mathbf{u}(t)\|_{L^{2}(\Omega)}^{2} \leq 4\left(\frac{h}{t}\right)^{2} \sum_{i=1}^{\infty} \sup _{\sigma \in[0, t+h]}\left|\left\langle\mathbf{f}(\sigma), \varphi_{i}\right\rangle\right|^{2} .
$$

Since (3.6), $\quad\|\mathbf{u}(t+h)-\mathbf{u}(t)\|_{L^{2}(\Omega)}^{2} \leq C\left(\frac{h}{t}\right)^{2} \rightarrow 0$ as $h \rightarrow 0$, and $\mathbf{u} \in$ $C\left((0, T] ; L^{2}(\Omega)\right)$.

IV) Finally, we analyze the asymptotic behavior of $\mathbf{u}(t)$ as $t \rightarrow 0$, assuming that (3.7) holds. From (3.7), for any $\varepsilon>0$ there exists a $\delta>0$ such that for 
any $0<t<\delta, \sum_{i=1}^{\infty} \sup _{\sigma \in[0, t]}\left|\left\langle\mathbf{f}(\sigma)-\mathbf{f}(0), \varphi_{i}\right\rangle\right|^{2}<\varepsilon$. Subtracting $\mathbf{u}(t)$ minus $u_{0}$ defined by (1.16), for any $0<t<\delta$, we deduce

$$
\begin{aligned}
\| \mathbf{u}(t) & -u_{0} \|_{H^{2}(\Omega)}^{2}=\sum_{i=1}^{\infty} \lambda_{i}^{2}\left[t^{-\lambda_{i}}\left(\int_{0}^{t} \sigma^{\lambda_{i}-1}\left\langle\mathbf{f}(\sigma), \varphi_{i}\right\rangle d \sigma\right)-\frac{1}{\lambda_{i}}\left\langle\mathbf{f}(0), \varphi_{i}\right\rangle\right]^{2} \\
& =\sum_{i=1}^{\infty} \lambda_{i}^{2}\left[t^{-\lambda_{i}}\left(\int_{0}^{t} \sigma^{\lambda_{i}-1}\left\langle\mathbf{f}(\sigma)-\mathbf{f}(0), \varphi_{i}\right\rangle d \sigma\right)\right]^{2} \\
& \leq \sum_{i=1}^{\infty} \lambda_{i}^{2} t^{-2 \lambda_{i}} \sup _{\sigma \in[0, t]}\left|\left\langle\mathbf{f}(\sigma)-\mathbf{f}(0), \varphi_{i}\right\rangle\right|^{2}\left(\int_{0}^{t} \sigma^{\lambda_{i}-1} d \sigma\right)^{2} \\
& =\sum_{i=1}^{\infty} \sup _{\sigma \in[0, t]}\left|\left\langle\mathbf{f}(\sigma)-\mathbf{f}(0), \varphi_{i}\right\rangle\right|^{2}<\varepsilon
\end{aligned}
$$

which concludes the proof.

\section{Acknowledgements}

Rosa Pardo was partially supported by Grant MTM2016-75465, Spain and Grupo de Investigación UCM 920894.

\section{References}

[1] J.R. Anderson. Local existence and uniqueness of solutions of degenerate parabolic equations. Comm. Partial Differential Equations, 16(1):105-143, 1991. https://doi.org/10.1080/03605309108820753.

[2] D. Andreucci. $L_{\mathrm{loc}}^{\infty}$-estimates for local solutions of degenerate parabolic equations. SIAM J. Math. Anal., 22(1):138-145, 1991. https://doi.org/10.1137/0522008.

[3] A.K. Aziz, D.A. French, S. Jensen and R. Bruce Kellogg. Origins, analysis, numerical analysis, and numerical approximation of a forward-backward parabolic problem. M2AN Math. Model. Numer. Anal., 33(5):895-922, 1999. https://doi.org/10.1051/m2an:1999125.

[4] D. Blanchard and G.A. Francfort. A few results on a class of degenerate parabolic equations. Ann. Scuola Norm. Sup. Pisa Cl. Sci. (4), 18(2):213-249, 1991.

[5] H. Brezis. Functional analysis, Sobolev spaces and partial differential equations. Universitext. Springer, New York, 2011.

[6] C.Xu, Z. Wang, C. Chang and F. Sun. Energy and exergy analysis of solar power tower plants. Applied Thermal Engineering, 31:3904-3913, 2011. Available from Internet: https://doi.org/10.1016/j.applthermaleng.2011.07.038.

[7] E. DiBenedetto, Y. Kwong and V. Vespri. Local space-analyticity of solutions of certain singular parabolic equations. Indiana Univ. Math. J., 40(2):741-765, 1991. https://doi.org/10.1512/iumj.1991.40.40033.

[8] L.C. Evans. Partial differential equations, volume 19 of Graduate Studies in Mathematics. American Mathematical Society, Providence, RI, second edition, 2010. https://doi.org/10.1090/gsm/019. 
[9] M.S. Floater. Blow-up at the boundary for degenerate semilinear parabolic equations. Arch. Rational Mech. Anal., 114(1):57-77, 1991. https://doi.org/10.1007/BF00375685.

[10] Y. Giga, S. Goto, H. Ishii and M.-H. Sato. Comparison principle and convexity preserving properties for singular degenerate parabolic equations on unbounded domains. Indiana Univ. Math. J., 40(2):443-470, 1991. https://doi.org/10.1512/iumj.1991.40.40023.

[11] V. Giovangigli. An existence theorem for a free boundary problem of hypersonic flow theory. SIAM J. Math. Anal., 24(3):571-582, 1993. https://doi.org/10.1137/0524035.

[12] J.A. Goldstein and Q.S. Zhang. On a degenerate heat equation with a singular potential. J. Funct. Anal., 186(2):342-359, 2001. https://doi.org/10.1006/jfan.2001.3792.

[13] D. Henry. Geometric theory of semilinear parabolic equations, volume 840 of Lecture Notes in Mathematics. Springer-Verlag, Berlin-New York, 1981.

[14] M. Ivanchov and N. Saldina. An inverse problem for strongly degenerate heat equation. Journal of Inverse and Ill-posed Problems, 14(5):465-480, 2019. https://doi.org/10.1515/156939406778247598.

[15] A.V. Ivanov. Uniform Hölder estimates for weak solutions of quasilinear doubly degenerate parabolic equations. Preprinty LOMI [LOMI Preprints], E10-89. Akad. Nauk SSSR, Mat. Inst. Leningrad. Otdel., Leningrad, 1989. https://doi.org/10.1007/BF01671935.

[16] O.V. Makhnei. Boundary problem for the singular heat equation. Carpathian Math. Publ., 9(1):86-91, 2017. https://doi.org/10.15330/cmp.9.1.86-91.

[17] I. Malyshev. On the parabolic potentials in degenerate-type heat equation. J. Appl. Math. Stochastic Anal., 4(2):147-160, 1991. https://doi.org/10.1155/S1048953391000114.

[18] K. Mochizuki and R. Suzuki. On blow-up of solutions for quasilinear degenerate parabolic equations. Publ. RIMS, Kyoto Univ., 745(27):193-201, 1991. Available from Internet: http://hdl.handle.net/2433/102185.

[19] O.A. Oleĭnik. The prandtl system of equations in boundary layer theory. Dokl. Akad. Nauk SSSR, 4(3):28-31, 1963.

[20] O.A. Oleĭnik. On the system of boundary-layer equations for axisymmetric flows. Dokl. Akad. Nauk SSSR, 175:77-80, 1967.

[21] N.H. Paul and J.R. Engel. Experience with the molten-salt reactor experiment. Nuclear Applications and Technology, 8(2):118-136, 1970. https://doi.org/10.13182/NT8-2-118.

[22] A. Pazy. Semigroups of linear operators and applications to partial differential equations, volume 44 of Applied Mathematical Sciences. Springer-Verlag, New York, 1983. https://doi.org/10.1007/978-1-4612-5561-1.

[23] T.N. Phuoc and L. Véron. Initial trace of positive solutions of a class of degenerate heat equation with absorption. Discrete Contin. Dyn. Syst., 33(5):2033-2063, 2013. https://doi.org/10.3934/dcds.2013.33.2033.

[24] M.M. Porzio. $L_{\text {loc }}^{\infty}$-estimates for degenerate and singular parabolic equations. Nonlinear Anal., 17(11):1093-1107, 1991. https://doi.org/10.1016/0362546X(91)90194-6. 
[25] P.A. Raviart and J.M. Thomas. Introduction à l'analyse numérique des équations aux dérivées partielles. Collection Mathématiques Appliquées pour la Maîtrise. [Collection of Applied Mathematics for the Master's Degree]. Masson, Paris, 1983.

[26] E.C. Young. Uniqueness of solution of a singular heat equation. Internat. J. Math. Math. Sci., $\mathbf{7}(1): 201-204,1984$. https://doi.org/10.1155/S0161171284000211.

[27] J.N. Zhao. Source-type solutions of degenerate quasilinear parabolic equations. J. Differential Equations, 92(2):179-198, 1991. https://doi.org/10.1016/00220396(91)90046-C.

[28] W. Zhou and P. Lei. A one-dimensional nonlinear heat equation with a singular term. J. Math. Anal. Appl., 368(2):711-726, 2010. https://doi.org/10.1016/j.jmaa.2010.03.066. 